\title{
Development of a small magnetic levitated centrifugal blood pump using a radial type self-bearing motor and axial position change of rotor-impeller by rotational magnetic field
}

\author{
Hiroyuki ONUMA*, Toru MASUZAWA** and Michiko MURAKAMI** \\ ${ }^{*}$ Department of Electronics and Control Engineering, National Institute of Technology, Ibaraki College \\ 866 Nakane, Hitachinaka, Ibaraki 312-8508, Japan \\ E-mail: onuma@ss.ibaraki-ct.ac.jp \\ ${ }^{* *}$ College of Engineering, Ibaraki University \\ 4-12-1 Nakanarusawa, Hitachi, Ibaraki 316-8511, Japan
}

Received: 30 January 2017; Revised: 2 May 2017; Accepted: 10 August 2017

\begin{abstract}
A small magnetic levitated centrifugal blood pump using a radial type self-bearing motor has been developed for use as an implantable artificial heart. In order to realize an implantable blood pump for a small adult patient, miniaturization and high efficiency of the device are necessary. In this study, a radial type self-bearing motor which is small-diameter and thin was developed, and the axial position change of the rotor-impeller by the rotational magnetic field was proposed. Magnetic suspension characteristics and motor performance were compared the center axial position of the rotor with the displaced axial position of the rotor. Additionally, a magnetic levitated centrifugal blood pump using the self-bearing motor was developed, and pump performance and levitation performance were measured. The magnetic suspension performance in the radial direction was enough ability to control the radial position of the rotor. The magnetic suspension force in the radial direction decreased by displacing the axial position of the rotor. The passive stability performance in the axial direction was enough ability to suspend the rotor. The restoring force was possible to be varied by the rotation magnetic field. The motor performance decreased by shifting the phase angle of the rotational magnetic field from 90 degrees and displacing the axial position of the rotor. At the operating condition with a flow rate of $5 \mathrm{~L} / \mathrm{min}$ against a pressure of $100 \mathrm{~mm} \mathrm{Hg}$, the oscillation amplitude in $\mathrm{x}, \mathrm{y}, \mathrm{z}$ direction were $0.014 \mathrm{~mm}, 0.014 \mathrm{~mm}$ and $0.039 \mathrm{~mm}$, respectively. And, the total power consumption was $7.1 \mathrm{~W}$. The developed magnetic levitated centrifugal blood pump has demonstrated sufficient levitation performance and low total power consumption. The average displacement in $\mathrm{z}$ direction of the rotor-impeller was possible to change by changing the phase angle of the rotational magnetic field. By decreasing the phase angle from 90 degrees in range of from 60 degrees to 90 degrees, it is possible to improve the levitation performance with just a little increases the total power consumption.
\end{abstract}

Keywords : Magnetic levitation, Magnetic bearing, Self-bearing motor, Magnetic levitated pump, Centrifugal blood pump, Ventricular assist device

\section{Introduction}

A heart transplant is one of a treatment for patient with severe heart failure. But, chronic donor shortage is serious problem. Most of the patients wait for a few years from a few months before receiving organ donation. Left ventricular assist devices (rotary blood pumps) that are mechanical devices for circulation have been researched and developed (Joyce, Joyce and Loebe, 2012). The mechanical life expectancy of a rotary blood pump is primarily determined by the durability of mechanical parts such as bearing and seals. Magnetic levitation technology is applied for durability enhancement of an artificial hearts (Merkel, et al., 2004, Murakami, et al., 2014, Nishinaka, et al., 2006, Onuma, et al., 
2014 and Osa, et al., 2014). The non-contacting behavior of the artificial hearts using the magnetic levitation technology is good for high durability, lower hemolytic properties, and anti-thrombogenesity. Centrifugal blood pumps using a radial type self-bearing motor were previously developed for use as an implantable artificial heart (Onuma and Masuzawa, 2014). Since the radial type self-bearing motor (Murakami, et al., 2014, Onuma, et al., 2015, Onuma, et al., 2014, Onuma, et al., 2012, Reichert, et al., 2012 and Silber and Amrhein, 1998) is able to generate a magnetic suspension force and a torque by one stator, it is suitable for miniaturization of the device. In the radial type self-bearing motor, the radial position and rotation of the rotor-impeller are actively controlled. And, the axial position and inclination of the rotor-impeller are passively suspended. In order to realize an implantable small centrifugal blood pump for a small adult patient, miniaturization, high efficiency and good levitation performance of the device are necessary. In our previous study, the combination of magnetic pole number of magnetic levitation's magnetic field and permanent magnet's magnetic field was examined by two-dimensional magnetic field analysis (Onuma, et al., 2012). As a result, it was found that the combination of a permanent magnet's magnetic field of pole number 8 and a magnetic levitation's magnetic field of pole number 6 is good pole number. Comparative experiments of radial type self-bearing motors with different magnetic levitation characteristics were carried out (Onuma, et al., 2014). In the paper, the design guidelines for the radial type self-bearing motor were suggested. The estimating equation of the restoring force corresponding to the radial type self-bearing motor's the passive stability in the axial direction was developed (Onuma, et al., 2015). And, in the paper, it was suggested that it is possible to control the restoring force in the axial direction by the rotational magnetic field. An axial vibration suppression method by d-axis current regulation was proposed (Miyoshi, et al., 2016). On the other hand, in a centrifugal pump, an axial thrust force acts on an impeller due to the pressure difference inside the pump. The rotor-impeller of the magnetic levitated centrifugal blood pump is displaced in the axial direction by the axial thrust force. The axial position change method of the rotor-impeller by the rotational magnetic field has not been proposed, yet. And, the influence of the axial displacement of the rotor on the characteristics of the radial type self-bearing motor has not yet been evaluated.

In this study, a radial type self-bearing motor which is small-diameter and thin was developed. And, the axial position change of the rotor-impeller by the rotational magnetic field was proposed. Then, the magnetic suspension characteristics and the motor performance were measured when the rotor displaced in the axial direction and the phase angle of the rotational magnetic field changed. Additionally, a magnetic levitated centrifugal blood pump using the self-bearing motor was developed. And, pump performance and levitation performance were measured. The axial position of the rotor-impeller in the magnetic levitated centrifugal blood pump was demonstrated to be changed by the rotational magnetic field.

\section{Methods}

\subsection{Magnetic levitated centrifugal blood pump}

Fig. 1 shows the schematic of the magnetic levitated centrifugal blood pump. The magnetic levitated centrifugal blood pump using the radial type self-bearing motor, which has the pump housing's the inner diameter of $48 \mathrm{~mm}$ and the pump housing's the thickness of $19.5 \mathrm{~mm}$, was developed. A closed type impeller with eight vanes was constructed on the top of the rotor. The outer diameter and the thickness of the rotor-impeller are $47 \mathrm{~mm}$ and $16.6 \mathrm{~mm}$, respectively. The mass of the rotor-impeller is $33 \mathrm{~g}$. Four eddy current sensors, which are differential sensing type sensors with opposing sensors, and three hall elements are used to control radial position and rotation speed of the rotor-impeller, respectively. Other four eddy current sensors are used to only measure the axial position and inclination of the rotor-impeller. Most narrow flow channel width in the radial direction and axial direction are $0.3 \mathrm{~mm}$ and $1.5 \mathrm{~mm}$, respectively. And, the rotor-impeller can be tilted geometrically 2.8 degrees in flow channel.

\subsection{Radial type self-bearing motor}

Fig. 2 shows the schematic illustration of the radial type self-bearing motor. The outer rotor structure, that a rotor is set around a stator, is adopted to miniaturize the device. The rotor has eight permanent magnets on inner circumferential surface. The stator set at the center of the pump has twelve teeth. The outer diameter of the stator is 35 $\mathrm{mm}$. The outer diameter of the rotor is $45.6 \mathrm{~mm}$. The thickness of the stator yoke and the rotor yoke is $5 \mathrm{~mm}$. The air gap between the stator and the rotor is $1.3 \mathrm{~mm}$. By most narrow flow channel width in the radial direction of pump 
casing, the rotor's mechanically suppressed range of movement in the radial direction is $0.3 \mathrm{~mm}$. By most narrow flow channel width in the axial direction of pump casing, the rotor's mechanically suppressed range of movement in the axial direction is $1.5 \mathrm{~mm}$. The radial position and rotation of the rotor-impeller are actively controlled through control of the magnetic field of the self-bearing motor. The axial position and inclination of the rotor-impeller are passively suspended by the strong magnetic attractive force in the radial direction between the stator and the rotor. The plus minus two-pole algorithm (Okada, et al., 1995) is adopted to levitate and rotate the rotor. Rotation coils of 50 turns and levitation coils of 80 turns are separately constructed in the each tooth of the stator. Rotation coils produce 3-phase 8-pole magnetic field. Levitation coils produce 2-phase 6-pole magnetic field.

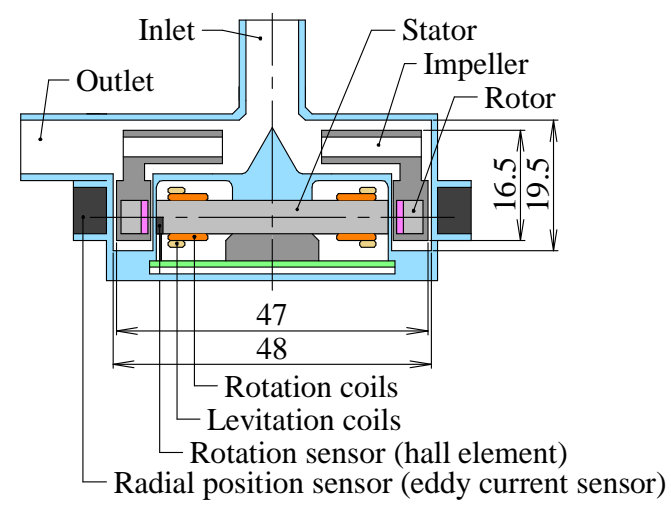

(a) Schematic illustration.

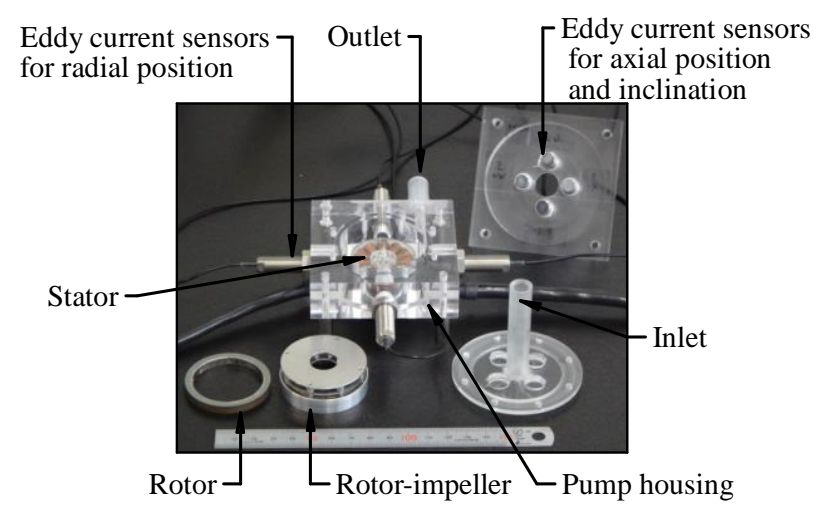

(b) Photograph of component parts.

Fig. 1 Schematic illustration of magnetic levitated centrifugal blood pump.

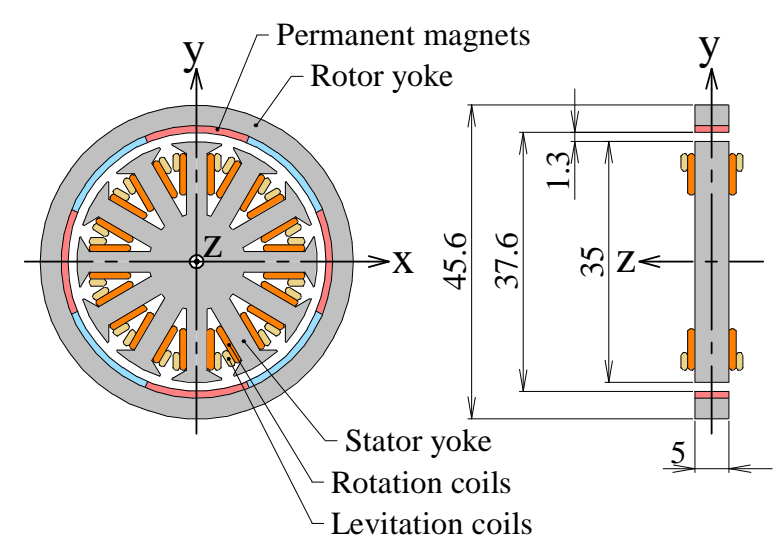

(a) Dimensional drawing.

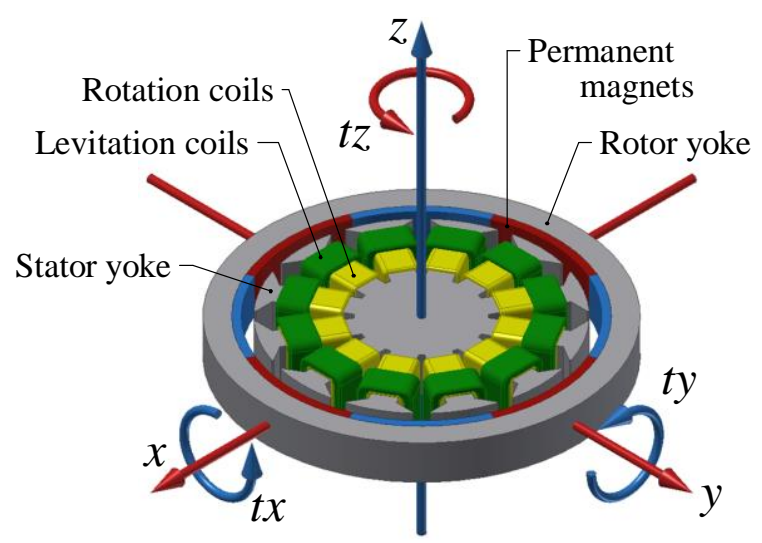

(b) Oblique perspective figure.

Fig. 2 Schematic illustration of radial type self-bearing motor.

\subsection{Experiments}

\subsubsection{Measurement of attractive force in radial direction}

The attractive force in the radial direction was measured using the load cell to evaluate the magnetic suspension force and negative spring force in the radial direction. The attractive force in radial direction measurement system is shown in Fig. 3. The rotor fixed to a holding fixture on a linear slider connected with a load cell on an x-stage. The stator fixed to a holding fixture connected with a base. The rotor was set at the center position of the rotor determined from the geometrical position of the holding fixture of the rotor and the stator. The amplitude of current for the levitation magnetic field was changed from $0 \mathrm{~A}$ to $3 \mathrm{~A}$. Current of $3 \mathrm{~A}$ is maximum current of power amplifier for control system of the radial type self-bearing motor. The rotor position in the radial direction was changed from $0 \mathrm{~mm}$ (center position) to $0.5 \mathrm{~mm}$ with micrometer of $\mathrm{x}$-stage. And, the rotor position in the axial direction was $0 \mathrm{~mm}$ (center position) and $1 \mathrm{~mm}$. 


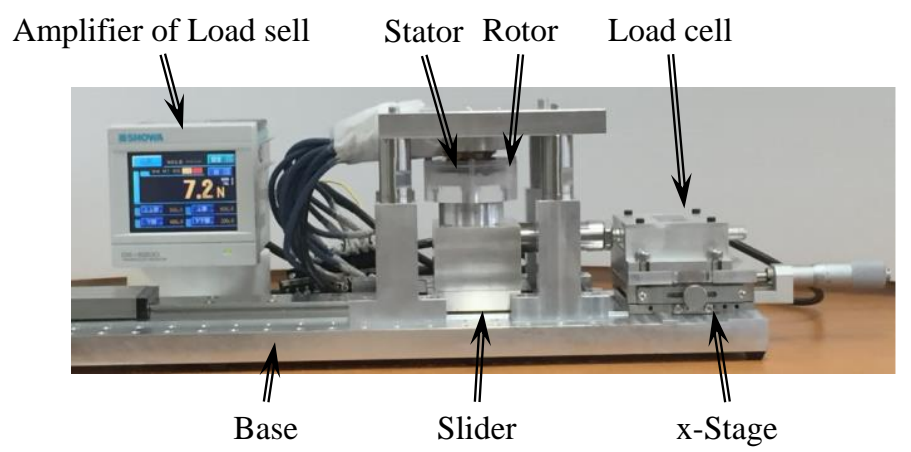

(a) Photo graph of measurement system.

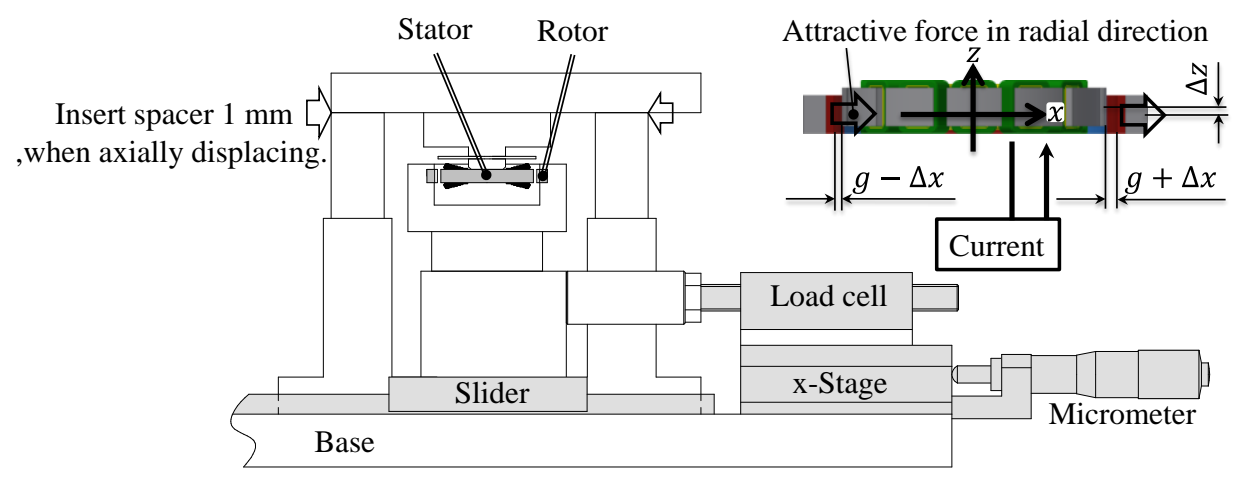

(b) Schematics of measurement system.

Fig. 3 Attractive force in radial direction measurement system.

\subsubsection{Measurement of Restoring Force in Axial Direction}

The attractive force in the axial direction as a restoring force was measured using the load cell to evaluate the passive stability performance in the axial direction. Additionally, the interference to the restoring force by the rotational magnetic field was measured. The attractive force in axial direction measurement system is shown in Fig. 4. The rotor fixed to a holding fixture on a linear slider connected with a load cell on an x-stage. The stator fixed to a holding fixture connected with a base. The rotor was set at the center position of the rotor determined from the geometrical position of the holding fixture of the rotor and the stator. The axial displacement of the rotor was changed from $0 \mathrm{~mm}$ to $5 \mathrm{~mm}$ with micrometer of $\mathrm{x}$-stage. The amplitude of current for the rotational magnetic field was changed from $0 \mathrm{~A}$ to $3 \mathrm{~A}$. Current of $3 \mathrm{~A}$ is maximum current of power amplifier for control system of the radial type self-bearing motor. And, the phase angle of the rotational magnetic field was set 0 degree, 90 degrees and 180 degrees. In a motor, the rotation control current is divided into d-axis component and q-axis component. The d-axis is the axis oriented toward the center of the permanent magnet. The q-axis is the axis oriented toward the direction of between permanent magnets. In this paper, the phase angle of the rotational magnetic field's control current is an angular difference in electrical angle from d-axis. So, relationship between the phase angle, the amplitude of current, d-axis component and q-axis component are represented by the following formulas.

$$
\begin{aligned}
& \mathrm{d} \text { - axis component }=(\text { amplitude of current }) \times \cos (\text { phase angle }) \\
& \mathrm{q} \text { - axis component }=(\text { amplitude of current }) \times \sin (\text { phase angle })
\end{aligned}
$$

Therefore, when the phase angle is 0 degree, it means the rotational magnetic field's control current has only positive $\mathrm{d}$-axis component. When the phase angle is 90 degree, it means the rotational magnetic field's control current has only q-axis component. When the phase angle is 180 degree, it means the rotational magnetic field's control current has only negative d-axis component. 


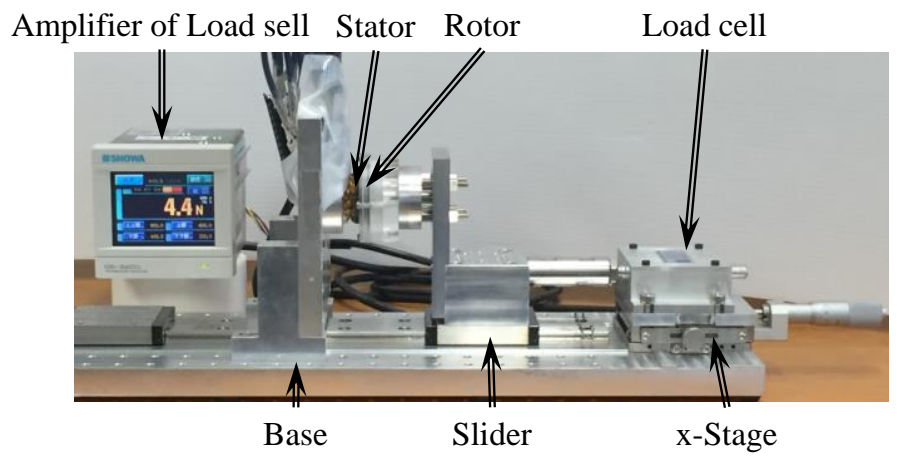

(a) Photo graph of measurement system.

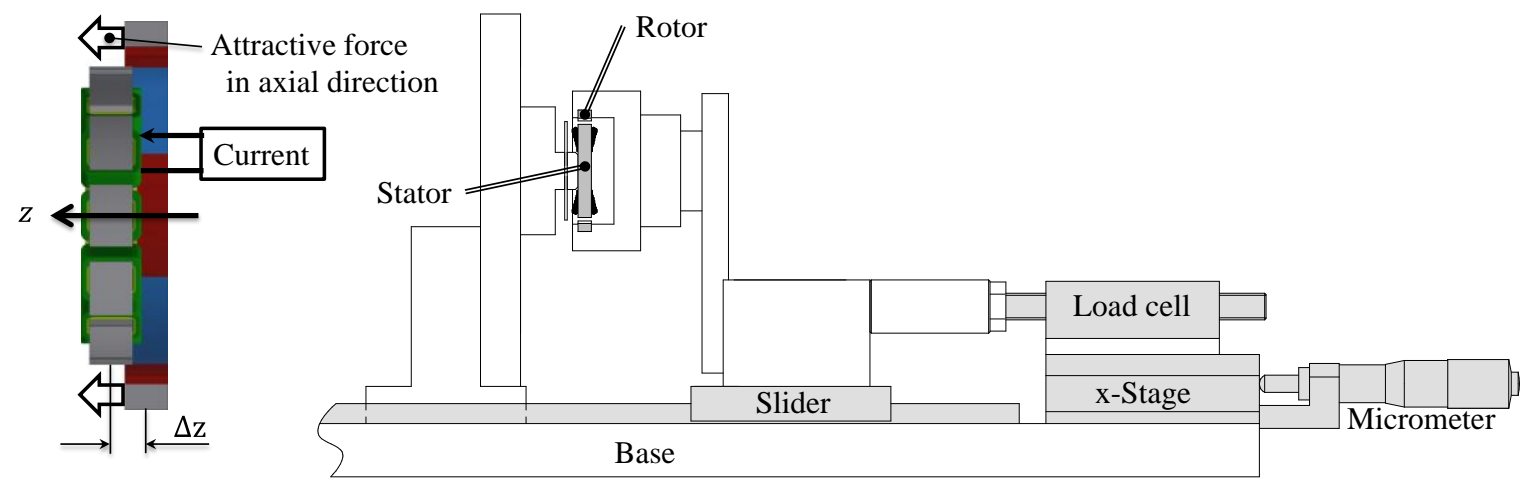

(b) Schematics of measurement system.

Fig. 4 Attractive force in axial direction measurement system.

\subsubsection{Measurement of Motor Performance}

The power consumption without load was measured to evaluate the motor performance at levitation in the air. The measurement system of motor performance at levitation is shown in Fig. 5. The average position and the oscillation amplitude of the rotor-impeller were measured to evaluate the levitation performance. Then, the electrical input power to the maglev pump was measured with the power meter. The rotational speed was set to $2,300 \mathrm{rpm}$. The phase angle of the rotational magnetic field was changed from 30 degree to 150 degrees. Rotation and levitation of the rotor-impeller were controlled with a digital PID controller using a digital signal processor. The parameters of the levitation PID controller were $\mathrm{K}_{\mathrm{lp}}=7 \mathrm{~A} / \mathrm{mm}, \mathrm{K}_{\mathrm{li}}=5 \mathrm{~A} / \mathrm{mm} / \mathrm{s}$ and $\mathrm{K}_{\mathrm{ld}}=0.016 \mathrm{~A} \cdot \mathrm{s} / \mathrm{mm}$ respectively. The parameters of the rotation PI controller were $\mathrm{K}_{\mathrm{rp}}=0.003 \mathrm{~A} / \mathrm{rpm}$ and $\mathrm{K}_{\mathrm{ri}}=0.7 \mathrm{~A} / \mathrm{rpm} / \mathrm{s}$, respectively.

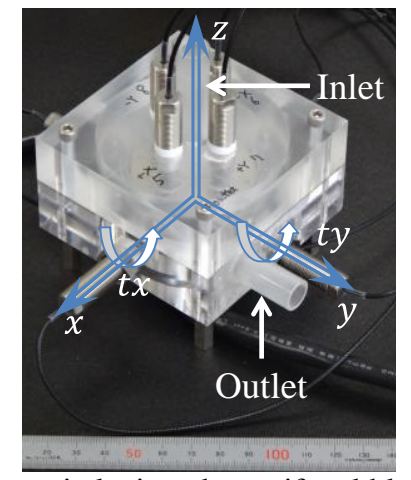

(a) Magnetic levitated centrifugal blood pump.

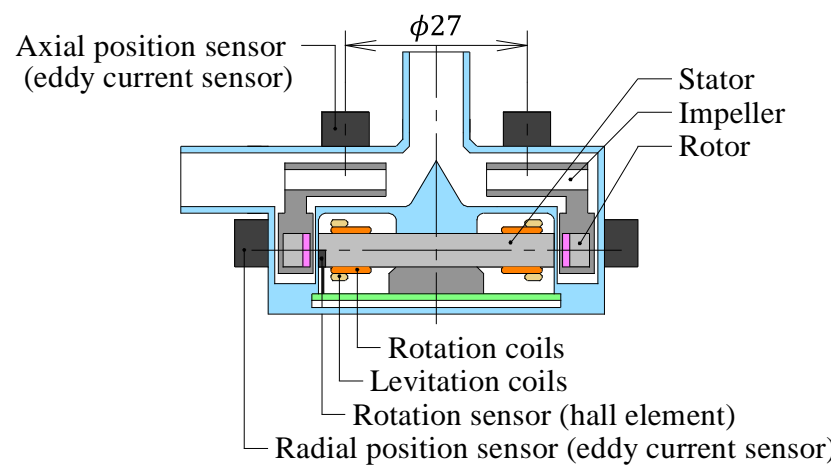

(b) Schematic of measurement system.

Fig. 5 Measurement system of motor performance at levitation.

The power consumption for rotation without load was measured to evaluate the motor performance change due to the phase angle for the rotational magnetic field and the axial position of the rotor. The motor performance measurement system is shown in Fig. 6. The rotor was attached to a shaft supported by ball bearings. The electrical 
input power to the motor was measured with the power meter. The rotational speed was set to 2,300 rpm. The phase angle of the rotational magnetic field was changed from 30 degree to 150 degrees. And, the rotor position in the axial direction was $0 \mathrm{~mm}$ (center position) and $1 \mathrm{~mm}$.

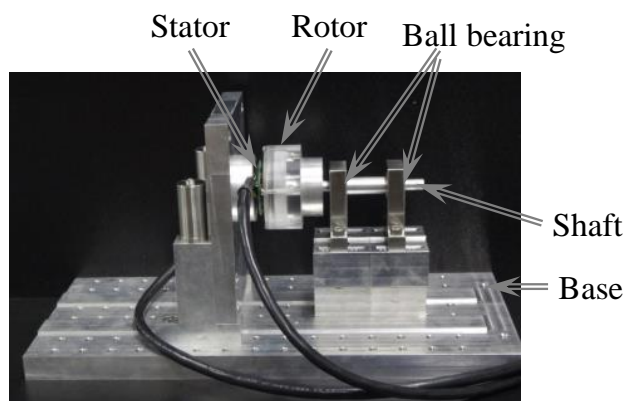

(a) Photo graph of measurement system.
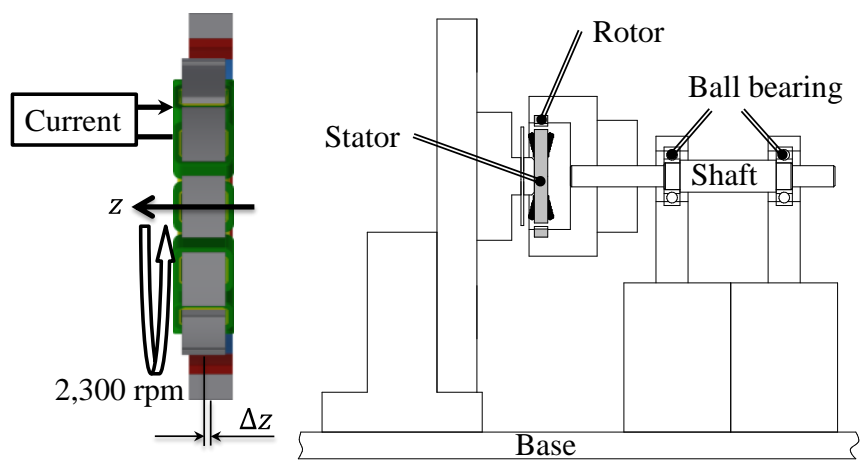

(b) Schematics of measurement system.

Fig. 6 Motor performance measurement system.

\subsubsection{Measurement of pump performance and levitation performance}

The magnetic levitated centrifugal blood pump was connected to a closed mock circuit filled with water, and pump and levitation performances were examined. The closed mock circuit is composed of two pressure gauge sensors, a flow meter, a reservoir tank, and a gate valve. The magnetic levitated centrifugal blood pump and the closed mock circuit are shown in Fig. 7. The average position and the oscillation amplitude of the rotor-impeller were measured to evaluate the levitation performance during pumping. Then, the electrical input power to the maglev pump was measured with the power meter. Rotation and levitation of the rotor-impeller were controlled with a digital PID controller using a digital signal processor. The parameters of the levitation PID controller were $\mathrm{K}_{\mathrm{lp}}=7 \mathrm{~A} / \mathrm{mm}, \mathrm{K} \mathrm{li}=5$ $\mathrm{A} / \mathrm{mm} / \mathrm{s}$ and $\mathrm{K}_{\mathrm{ld}}=0.016 \mathrm{~A} \cdot \mathrm{s} / \mathrm{mm}$ respectively. The parameters of the rotation PI controller were $\mathrm{K}_{\mathrm{rp}}=0.003 \mathrm{~A} / \mathrm{rpm}$ and $\mathrm{K}_{\mathrm{ri}}=0.7 \mathrm{~A} / \mathrm{rpm} / \mathrm{s}$, respectively. The phase angle of rotational magnetic field and permanent magnet's field was 90 degrees.

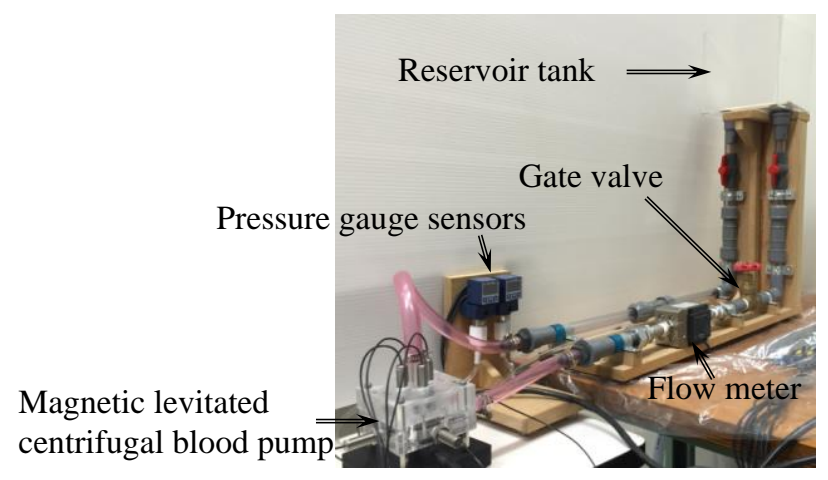

Fig. 7 Measurement system of pump performance and levitation performance.

\subsubsection{Measurement of the axial position change caused by rotational magnetic field}

The effect to the axial position of the rotor-impeller by the rotational magnetic field was evaluated with the system of Fig. 7 which measured the pump performance and levitation performance. Rotational speed was set to 2,300 rpm to achieve a flow rate of $5 \mathrm{~L} / \mathrm{min}$ against a pressure of $100 \mathrm{mmHg}$. The phase angle of the rotational magnetic field was changed from 30 degrees to 150 degrees. The average position and the oscillation amplitude of the rotor-impeller were measured to evaluate the levitation performance during pumping. Then, the electrical input power to the maglev pump was measured with the power meter. 


\section{Experimental results and discussion 3.1 Attractive Force in Radial Direction}

The measured attractive force in the radial direction is shown in Fig. 8. The magnetic suspension force of axial position $0 \mathrm{~mm}$ and $1 \mathrm{~mm}$ generated by a current of $3 \mathrm{~A}$ at radial displacement of $0 \mathrm{~mm}$ were $6.9 \mathrm{~N}$ and $6.5 \mathrm{~N}$, respectively. When the rotor moved $1 \mathrm{~mm}$ in the axial direction, the magnetic suspension force decreased $6 \%$. The negative spring force of axial position $0 \mathrm{~mm}$ and $1 \mathrm{~mm}$ generated by displacement of $0.5 \mathrm{~mm}$ was $-6.1 \mathrm{~N}$ and $-6.0 \mathrm{~N}$, respectively.

The magnetic suspension force that can be generated by the levitation magnetic field is sufficiently stronger than the negative spring force generated by the rotor displacement. The developed self-bearing motor has position control ability of the rotor movement range of $0.5 \mathrm{~mm}$ or more. The rotor's mechanically suppressed range of movement in the radial direction is $0.3 \mathrm{~mm}$. And, the developed self-bearing motor is able to return the rotor at a radial displacement of $0.3 \mathrm{~mm}$ to the radial center position with a current of $1.5 \mathrm{~A}$. Therefore, it has very good control ability for the radial position of the rotor. When the rotor moved in the axial direction, the magnetic suspension force in the radial direction decreased. This reason is that the generation efficiency of the magnetic suspension force in the radial direction reduced by decreasing the facing area between the rotor and the stator. The axial displacement of the rotor is not preferred for radial control.

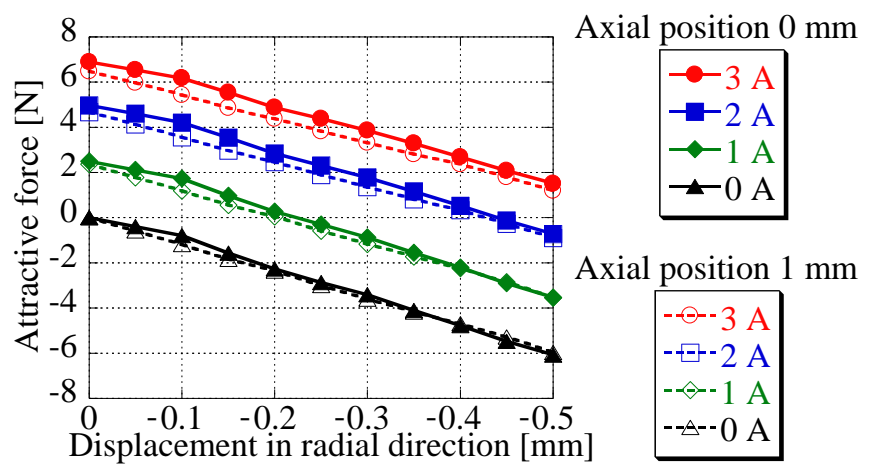

Fig. 8 Attractive force in the radial direction.

\subsection{Restoring Force in Axial Direction}

The measured restoring force in the axial direction with respect to each displacement in the axial direction when was given a current of $0 \mathrm{~A}$ and a current of $1 \mathrm{~A}$ which changed a phase angle is shown in Fig. 9 (a). The measured restoring force in the axial direction at a displacement of $1 \mathrm{~mm}$ with respect to each current which changed a phase angle is shown in Fig. 9 (b). The restoring force at the current of 0 A and a displacement of $1 \mathrm{~mm}$ was $2.7 \mathrm{~N}$. The maximum of the restoring force was $6.0 \mathrm{~N}$ at the displacement of $3.5 \mathrm{~mm}$.

The rotor-impeller mass is $33 \mathrm{~g}$. If the axial direction of the radial type self-bearing motor is opposite to the direction of gravity, the axial displacement of the rotor is $0.12 \mathrm{~mm}$. The rotor's mechanically suppressed range of movement in the axial direction is $1.5 \mathrm{~mm}$. Therefore, the passive stability performance in the axial direction was enough ability to suspend the rotor-impeller. The restoring force of a phase angle of 90 degrees (only q-axis component) was the almost same as it at a current of $0 \mathrm{~A}$. When the phase angle was less than 90 degrees (add d-axis component), the restoring force became stronger in proportion to the current than it a current of 0 A. When the phase angle was larger than 90 degrees (add d-axis component as a negative current), the restoring force became weaker in proportion to the current than it at a current of $0 \mathrm{~A}$. It has been found to be varied a restoring force by the rotational magnetic field. Furthermore, it was found that the restoring force can be controlled mainly by the d-axis component. When the permanent magnet's field and the rotational magnetic field were facing at different magnetic poles, the restoring force was increased by increasing the magnetic flux density in the air gap. Conversely, when the permanent magnet's field and the rotational magnetic field were facing at same magnetic poles, the restoring force was decreased by decreasing the magnetic flux density in the air gap. 


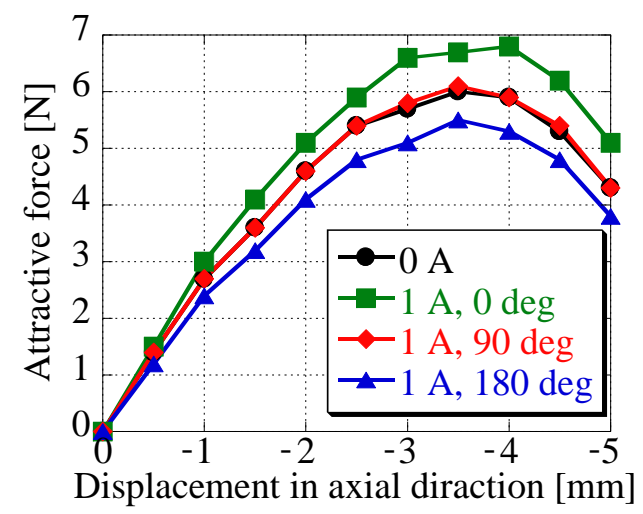

(a) Current of 0 A and $1 \mathrm{~A}$.

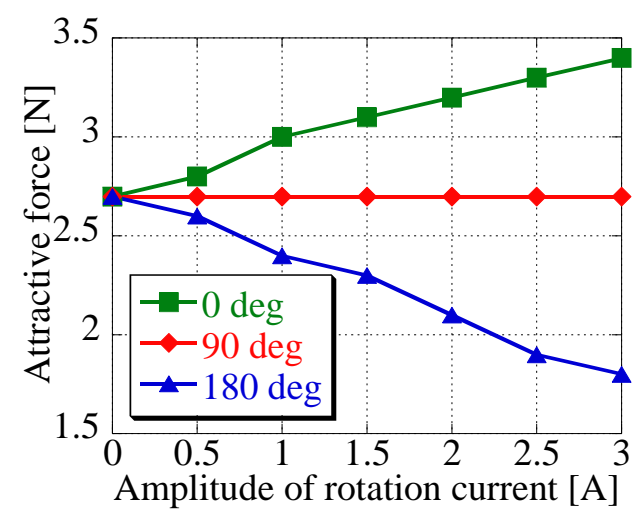

(b) Displacement of $1 \mathrm{~mm}$.

Fig. 9 Restoring force in axial direction.

\subsection{Motor performance}

Fig. 10 shows the power consumption at levitation and the power consumption for rotation without load at $0 \mathrm{~mm}$ and $1 \mathrm{~mm}$ of the axial position of the rotor when the rotational speed is $2,300 \mathrm{rpm}$. In the air, the average displacement in $\mathrm{x}, \mathrm{y}$ and $\mathrm{z}$ direction of the rotor-impeller were $0.0003 \mathrm{~mm}, 0.0005 \mathrm{~mm}$ and $-0.13 \mathrm{~mm}$, respectively. The oscillation amplitude in $\mathrm{x}, \mathrm{y}$ and $\mathrm{z}$ direction of the rotor-impeller were $0.017 \mathrm{~mm}, 0.017 \mathrm{~mm}$ and $0.020 \mathrm{~mm}$, respectively. The power consumption at levitation was $0.71 \mathrm{~W}$. The power consumption at levitation includes the power consumption for levitation and the power consumption for rotation. The power consumption for levitation was $0.39 \mathrm{~W}$. The power consumption for levitation was $0.32 \mathrm{~W}$. The power consumption at levitation did not change even if the phase angle was changed. In the measurement of motor performance when the rotor was attached to a shaft supported by ball bearings, at the phase angle is 90 degrees, the power consumption for rotation at $0 \mathrm{~mm}$ and $1 \mathrm{~mm}$ of the axial position of the rotor were $3.5 \mathrm{~W}$ and $3.9 \mathrm{~W}$, respectively. At the axial position of the rotor is $1 \mathrm{~mm}$, the power consumption for rotation increased $11 \%$. When the phase angle was shifted from 90 degrees, the power consumption for rotation increased. When the phase angle shifted from 90 degrees to 60 degrees and 120 degrees, the power consumption for rotation increased 5\%. When the phase angle shifted to 30 degrees and 150 degrees, the power consumption for rotation sharply increased $50 \%$.

In without load condition at levitation, since the power consumption for rotation is very low, it seems that it could not detect the power consumption change even if the phase angle was changed. In the measurement of motor performance when the rotor was attached to a shaft supported by ball bearings, since the loss in ball bearings is large, it seems that the power consumption change appears when the phase angle is changed. When the rotor moved in the axial direction, the power consumption for rotation increased. This reason is that the generation efficiency of the motor torque reduced by decreasing the facing area between the rotor and the stator. The axial displacement of the rotor is not preferred for motor performance. It is known that the motor generates torque in proportion to $\sin (\theta)$ where $\theta$ is the phase angle (A. E. Fitzgerald, et al., 2002). Therefore, at the phase angle is 90 degrees, the maximum torque generates.

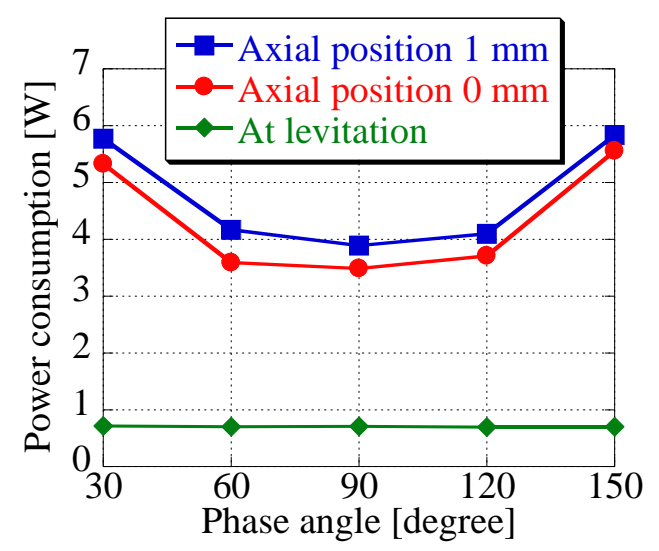

Fig. 10 Power consumption without load. 
And, it seems that the power consumption for the rotation increases a little bit at 60 degrees and 120 degrees, but increases sharply at 30 degrees and 150 degrees.

\subsection{Pump performance and levitation performance}

Fig. 11 shows HQ characteristics. The pump was levitated and rotated up to a rotating speed of 3,000 rpm successfully. The maximum pressure and the maximum flow rate were approximately $203 \mathrm{~mm} \mathrm{Hg}$ and $9.8 \mathrm{~L} / \mathrm{min}$, respectively. The target pump performance which is a flow rate of $5 \mathrm{~L} / \mathrm{min}$ against a pressure of $100 \mathrm{mmHg}$ is achieved with a rotating speed of 2,300 rpm. At a low flow rate, the bottom surface of the rotor-impeller was in contacted with the pump housing. At a rotating speed of 2,000 rpm, it was in contacted when a flow rate is $0 \mathrm{~L} / \mathrm{min}$. At a rotating speed of 2,300 rpm, it was in contacted when a flow rate is $1 \mathrm{~L} / \mathrm{min}$ or less. At a rotating speed of 2,500 rpm, it was in contacted when a flow rate is $2 \mathrm{~L} / \mathrm{min}$ or less. At a rotating speed of 3,000 rpm, it was in contacted when a flow rate is $4 \mathrm{~L} / \mathrm{min}$ or less. Fig. 12 shows the relationship between the total power consumption and the flow rate. The maximum total power consumption was $21.5 \mathrm{~W}$ at a rotating speed of 3,000 rpm and a flow rate of $9.8 \mathrm{~L} / \mathrm{min}$. The total power consumption for the target pump performance was $7.1 \mathrm{~W}$. Fig. 13 shows the average displacement and average tilt angle of the rotor-impeller which depend on each flow rate at a rotating speed of 2,300 rpm. At a flow rate of $5 \mathrm{~L} / \mathrm{min}$, average displacement in $\mathrm{x}, \mathrm{y}$ and $\mathrm{z}$ direction were $-0.0004 \mathrm{~mm}, 0.0006 \mathrm{~mm}$ and $-0.49 \mathrm{~mm}$, respectively. And, average tilt angle in tx and ty direction were -0.29 degree and 0.17 degree, respectively. Fig. 14 shows the oscillation amplitude in $\mathrm{x}, \mathrm{y}, \mathrm{z}$ direction and the oscillation amplitude in $\mathrm{tx}$ and ty direction of the rotor-impeller which depend on each flow rate at a rotational speed of $2300 \mathrm{rpm}$. At a flow rate of $5 \mathrm{~L} / \mathrm{min}$, oscillation amplitude in $\mathrm{x}, \mathrm{y}$ and $\mathrm{z}$ direction were $0.014 \mathrm{~mm}, 0.014 \mathrm{~mm}$ and $0.039 \mathrm{~mm}$, respectively. And, oscillation amplitude in tx and ty direction were 0.34 degree and 0.37 degree, respectively.

The target pump performance is a flow rate of $5 \mathrm{~L} / \mathrm{min}$ against a pressure of $100 \mathrm{mmHg}$. We believe that the total power consumption of a left ventricular assist device suitable for implantation should be less than $15 \mathrm{~W}$ with a flow rate of $5 \mathrm{~L} / \mathrm{min}$ against a pressure of $100 \mathrm{~mm} \mathrm{Hg}$. At the operating condition to generate the target pump performance, the developed magnetic levitated centrifugal blood pump has demonstrated sufficient levitation performance and low total power consumption. At a low flow rate, the average displacement in $\mathrm{z}$ direction and the oscillation amplitude were increased. Then, the bottom surface of the rotor-impeller was in contacted with the pump housing. The reason is that the thrust force in the axial direction increased with operating conditions approaching the shut off operation of the flow. It is possible to reduce the thrust force in the axial direction by boring the balance holes on the shroud of the impeller. When the rotor-impeller contacted with the pump housing, the total power consumption increased. It is because the power consumption for rotation was significantly increased by friction of the rotor-impeller's bottom surface and the pump housing. In order to further reduce the power consumption, if zero power control (Masuzawa, et al., 2004) is applied to the radial magnetic levitation control, it is possible to reduce the power consumption for levitation.

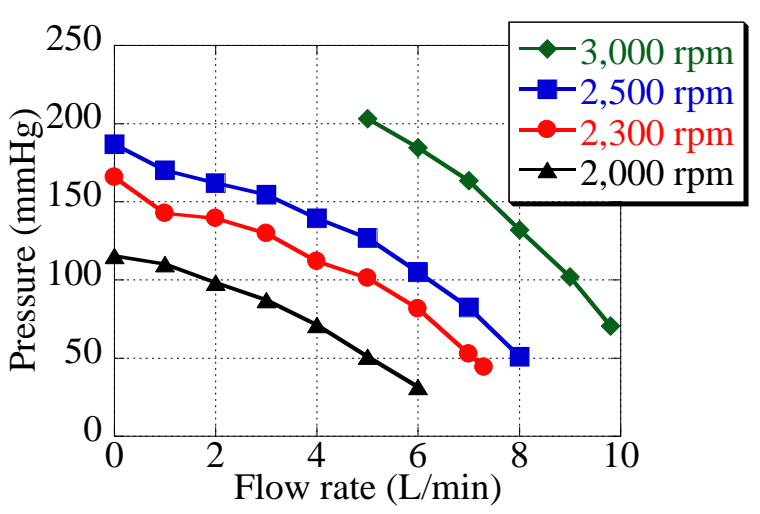

Fig. 11 HQ characteristics.

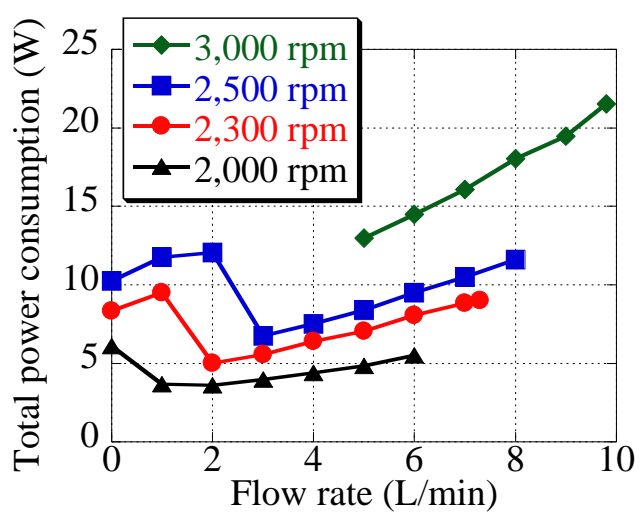

Fig. 12 Total power consumption versus flow rate. 


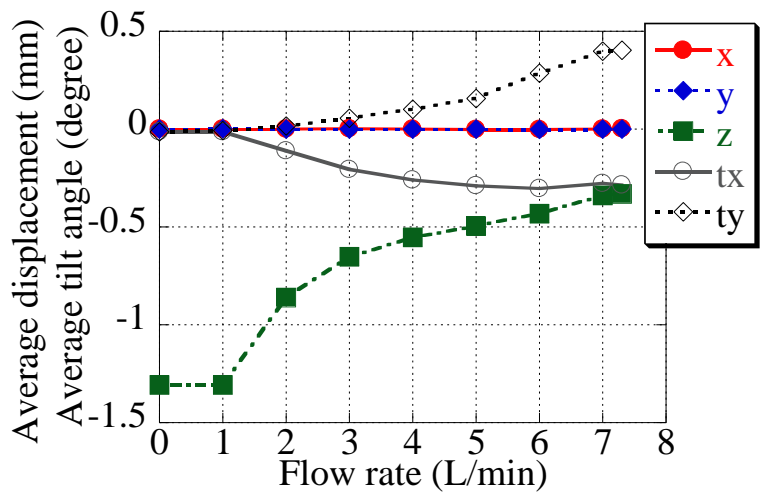

Fig. 13 Average displacement and average tilt angle versus flow rate at a rotating speed of 2,300 rpm.

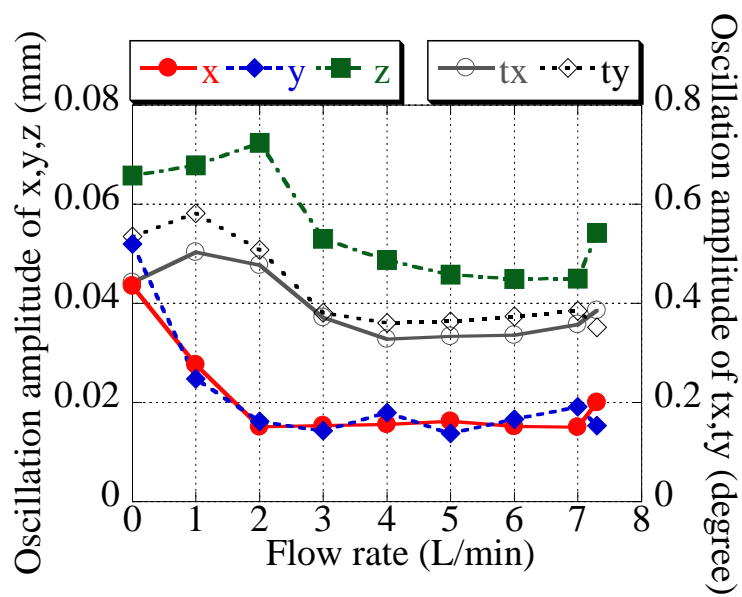

Fig. 14 Oscillation amplitude versus flow rate at a rotating speed of $2,300 \mathrm{rpm}$.

\subsection{The axial position change caused by rotational magnetic field}

At a rotating speed of 2,300 rpm, the average displacement and the average tilt angle of the rotor-impeller which depend on the phase angle of the rotational magnetic field is shown in Fig. 15. The average displacement in $\mathrm{x}$ and $\mathrm{y}$ direction, and the average tilt angle in tx and ty direction caused little or no change by changing the phase angle. The average displacement in $\mathrm{z}$ direction increased downward with the increasing the phase angle. Fig. 16 shows the oscillation amplitude of the rotor-impeller which depend on the phase angle at a rotational speed of 2,300 rpm. The oscillation amplitude in $\mathrm{x}$ and $\mathrm{y}$ direction caused little or no change by changing the phase angle. The oscillation amplitude in $\mathrm{z}, \mathrm{tx}$ and ty direction increased with the increasing the phase angle. The power consumption for rotation and levitation at a rotational speed of $2,300 \mathrm{rpm}$ is shown in Fig. 17. When the phase angle was shifted from 90 degrees, the power consumption for rotation increased. The power consumption for levitation increased with the increasing the phase angle. The d-axis component and the q-axis component of rotation current is shown in Fig. 18. The $\mathrm{d}$-axis component was $0 \mathrm{~A}$ at the phase angle of 90 degrees. When the phase angle was smaller than 90 degrees, the $\mathrm{d}$-axis component was increased in the positive direction. When the phase angle was larger than 90 degrees, the $\mathrm{d}$-axis component was increased in the negative direction. The q-axis component did not change even if the phase angle was changed.

It was possible to change the average displacement in $\mathrm{z}$ direction of the rotor-impeller by changing the phase angle of the rotational magnetic field. The reason why the q-axis component did not change is that the same torque is required to perform the same pump work at the same rotation speed. When the phase angle was smaller than 90 degrees, the average displacement in $\mathrm{z}$ direction and the oscillation amplitude in $\mathrm{z}$ direction were decreased. It is because the restoring force was increased. When the phase angle was shifted from 90 degrees, the power consumption for rotation increased. And, the power consumption for rotation at the phase angle of 150 degrees was higher than it at the phase angle of 30 degrees. This reason is that the generation efficiency of the motor torque reduced by decreasing the facing area between the rotor and the stator. The displacement in the axial direction of the rotor at the phase angle of 150 degrees is larger than it at the phase angle of 30 degrees. When the phase angle is smaller than 90 degrees, the power consumption for rotation is increased. But, the power consumption for levitation is decreased. It seems that the axial displacement is affecting the generation efficiency of the magnetic suspension force in the radial direction. The generation efficiency of the magnetic suspension force in the radial direction was improved by reducing the axial displacement of the rotor and the stator. Therefore, the power consumption for levitation is decreased. By decreasing the rotational magnetic field's the phase angle from 90 degrees in range of from 60 degrees to 90 degrees, it is possible to improve the levitation performance with just a little increases the total power consumption. In this paper, we investigated by focusing on the phase angle which is the parameter used for controlling the rotational magnetic field. In the rotational control system using the amplitude of current for the rotational magnetic field and the phase angle used in the magnetic levitated centrifugal blood pump, the restoring force may fluctuate with the fluctuation of the rotational 
torque by the rotation control. Since the restoring force can be controlled mainly by the d-axis component, it is possible to improve the control of the restoring force by changing to the rotational control system using the d-axis component and q-axis component. Thereby, the levitation performance might be further improved.

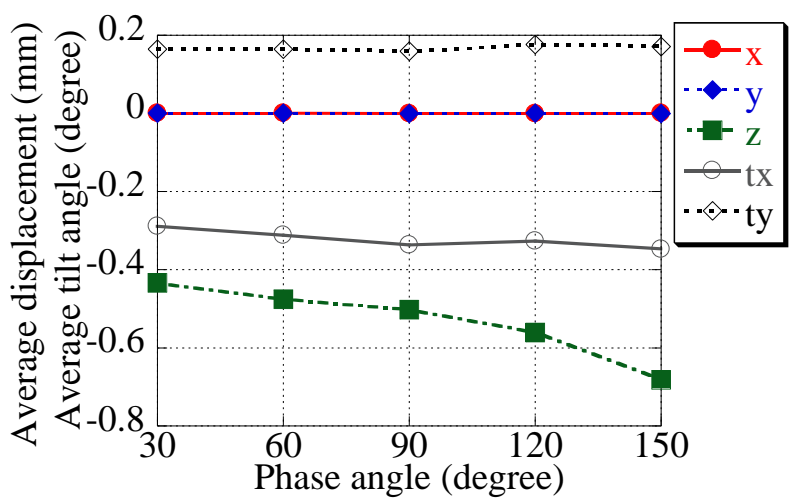

Fig. 15 Average displacement and average tilt angle versus phase angle.

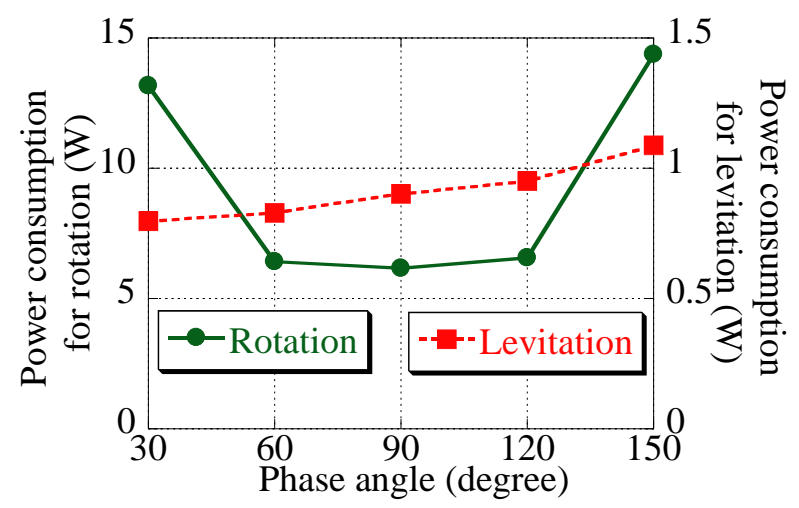

Fig. 17 Power consumption for rotation and levitation versus phase angle.

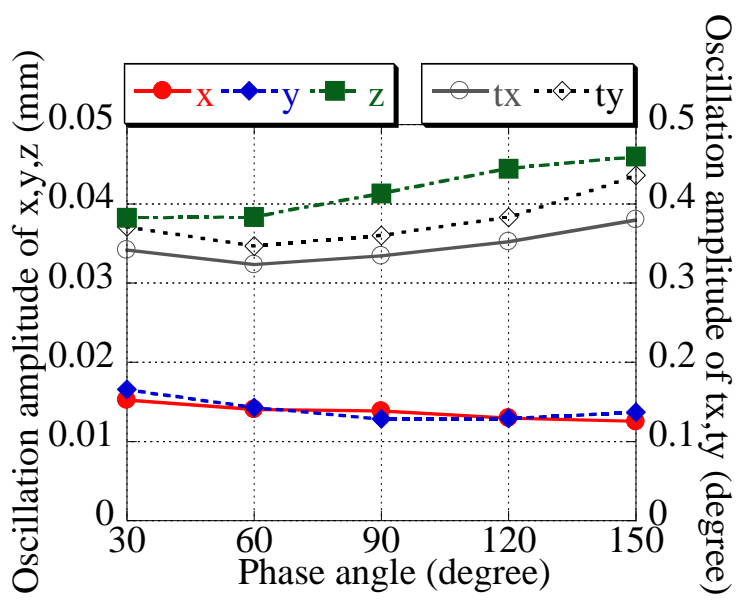

Fig. 16 Oscillation amplitude versus phase angle.

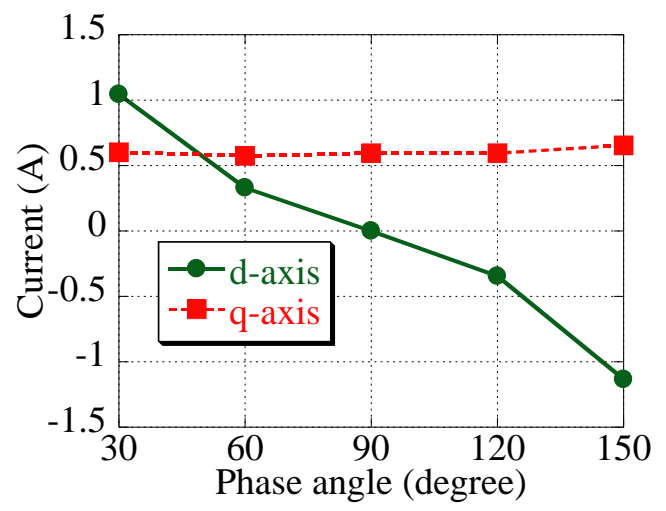

Fig. 18 d-axis component and q- axis component of rotation current versus phase angle.

\section{Conclusion}

The small radial type self-bearing motor was developed, and magnetic suspension characteristics and motor performance were measured. The developed self-bearing motor has very good radial position control ability of the rotor movement range of $0.5 \mathrm{~mm}$ or more. And, it was confirmed that the magnetic suspension force in the radial direction decreased by shifting the rotor in the axial direction. The passive stability performance in the axial direction was enough ability to suspend the rotor-impeller. The restoring force was possible to be varied by the rotational magnetic field. The power consumption for rotation without load was increased when the phase angle for the rotational magnetic field was shifted from 90 degrees or the rotor moved in the axial direction. The small magnetic levitated centrifugal blood pump using the self-bearing motor was developed. At the operating condition with a flow rate of $5 \mathrm{~L} / \mathrm{min}$ against a pressure of $100 \mathrm{~mm} \mathrm{Hg}$, the developed small magnetic levitated centrifugal blood pump has demonstrated sufficient levitation performance and low total power consumption. The average displacement in $\mathrm{z}$ direction of the rotor-impeller was possible to change by changing the phase angle of the rotational magnetic field. By decreasing the rotational magnetic field's the phase angle from 90 degrees in range of from 60 degrees to 90 degrees, it is possible to improve the levitation performance with just a little increases the total power consumption. This phase angle range is effective to reduce the axial displacement and the axial oscillation of the rotor-impeller. 


\section{Acknowledgment}

This work was supported by JSPS KAKENHI Grant Number 25820077.

\section{References}

A. E. Fitzgerald, Charles Kingsley, Jr. and Stephen D. Umans, Electric Machinery Sixth Edition (2002), Mc Graw Hill. Joyce, D. L., Joyce, L. D. and Loebe, M., Mechanical Circulatory Support Principles and Applications, Mc Graw Hill Medical (2012).

Masuzawa, T., Onuma, H. and Okada, Y., Zero Power Control for Magnetically Suspended Artificial Heart, Proceedings of 47th Proceedings of the Japan Joint Automatic Control Conference (2004), 322. (in Japanese)

Miyoshi, M., Sugimoto, H. and Chiba, A., Axial Vibration Suppression by Field Flux Regulation in Two-Axis Actively Positioned Permanent Magnet Bearingless Motors with Axial Position Estimation, Proceedings of 2016 XXII International Conference on Electrical Machines (2016), pp. 2574-2580.

Merkel, T., Amdt, A., Hoffmann, J., Nusser, P., Graichen, K., Neumann, W. and Muller, J., Magnetic bearing in INCOR axial blood pump acts as multifunctional sensor, Proceedings of 9th International Symposium on Magnetic Bearings (2004), \#33 on CDROM.

Murakami, M., Masuzawa, T., Yoshida, S., Onuma, H., Nishimura, T. and Kyo, S., Thin Maglev Ventricular Assist Device with Radial Type Self-bearing Motor, Proceedings of 14th nternational Symposium on Magnetic Bearings (2014), pp. 168-173.

Nishinaka, T., Schima, H., Roethy, W., Rajek, A., Nojiri, C., Wolner, E. and Wieselthaler, G M, The DuraHeart VAD, a Magnetically Levitated Centrifugal pump: The University of Vienna Bridge-to-transplant Experience, Circulation journal, vol. 70, no. 11 (2006), pp. 1421-1425.

Okada, Y., Ohishi, T. and Dejima, K., General Solution of Levitation Control of a Permanent Magnet (PM) -Type Rotating Motor, JSME International Journal Series C, vol. 38, no. 3 (1995), pp.538-542.

Onuma, H., Masuzawa, T. and Murakami, M., Estimating equation of the radial type self-bearing motor's the passive stability, Proceedings of 24th MAGDA Conference in Tohoku (2015), pp.53-58. (in Japanese)

Onuma, H. and Masuzawa, T., Evaluation of Magnetic Suspension Characteristics and Levitation Performance of A Centrifugal Blood Pump Using Radial Type Self-Bearing Motor, Proceedings of 14th International Symposium on Magnetic Bearings (2014), pp. 174-179.

Onuma, H., Ukita, K. and Masuzawa, T., Optimum pole number of a radial type self-bearing motor using 12 slots stator for artificial heart, Journal of JSAEM, vol. 20, no. 1 (2012), pp.59-65. (in Japanese)

Osa, M., Masuzawa, T., Omori, N. and Tatsumi, E., Radial Position Active Control of Double Stator Axial Gap Self-bearing Motor for Paediatric VAD, Proceedings of 14th International Symposium on Magnetic Bearings (2014), pp.187-192.

Reichert, T., Nussbaumer, T. and Kolar, J. W., Bearingless 300-W PMSM for Bioreactor Mixing, IEEE Transactions on industrial electronics, vol. 59, no. 3 (2012), pp. 1376-1388.

Silber, S. and Amrhein, W., Bearingless single-phase motor with concentrated full pitch windings in exterior rotor design, Proceedings of 6th International Symposium on Magnetic Bearings (1998), pp. 476-485. 